\title{
Severe Hypoglycaemic Events and CV Outcomes in TECOS: Bidirectional Association Indicating a 'Frail' Patient Phenotype
}

Standl E, ${ }^{1}$ Stevens S, ${ }^{2}$ Armstrong PW, ${ }^{3}$ Buse JB, ${ }^{4}$ Green JB, ${ }^{2}$ Lachin JM,,${ }^{5}$ Van de Werf F, ${ }^{6}$ Peterson ED, ${ }^{2}$ Holman RR, ${ }^{7}$ for the TECOS Study Group

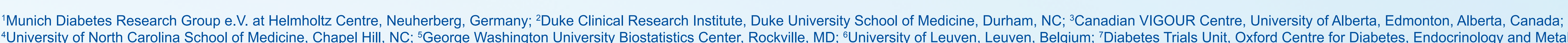

\section{Introduction}

Several large-scale long-term randomised cardiovascular (CV) outcome trials evaluating glucose-lowering drugs or strategies have identified an association between mortality or CV mortality in people with type 2 diabetes [1-5. This is particularly the case for patients experiencing severe hypoglycaemic events (SHEs) where a two-fold higher risk of $\mathrm{CV}$ death is suggested as compared with those withou SHE(s). However, it remains unclear whether SHEs have an association with increasing $\mathrm{CV}$ mortality rates. Hence exploring the tradeoff between adopting more cautious glycaemic targets to minimise $\mathrm{CV}$ risk with the consequen likelihood of an increased risk of microvascular disease [6] is warranted.

\section{Objectives}

We conducted post-hoc analyses of the Trial Evaluating (TECOS) [7] to better elucidate the relationship between SHEs and subsequent $\mathrm{CV}$ events as well as $\mathrm{CV}$ events and subsequent SHEs. CV outcomes were collected assiduous in TECOS and independently adjudicated, along with Pe special interst. TECOS enrolled 14,671 participants with type 2 diabetes and $\mathrm{CV}$ disease and followed them for

\section{Methods}

Ime-dependent associations between SHEs and prespecified primary and secondary clinical CV outcomes (see examined bidirectionally.

SHEs

All SHEs were recorded systematically as pre-specified events of clinical interest. They were defined per protocol as or incapacitated as partich help from individual or from medical personn tie third an assistance (irrespective of whether this assistance was actually provided).

Clinical Outcomes

The TECOS primary composite outcome was a 4-poin major adverse cardiovascular event (MACE), defined as the first confirmed event of $\mathrm{CV}$ death, nonfatal myocardia infarction (MI), nonfatal stroke, or hospitalization for unstable angina. Other TECOS secondary outcomes included 3-point MACE (CV death, nonfatal MI or nonfatal stroke), fatal/ nonfatal MI, fatal/nonfatal stroke, all-cause death and hospitalization for heart failure (hHF).

Statistical Analysis

To investigate the association between SHEs and subsequent CV or mortally oulcomes, Cox regression models were fitted with SHE as a time-dependent variable. Monels were repeated with partial adjustment for selected clinical factors (age, sex, race, smoking, and weight) and Win full adus (age, sex, race, smoking, and weigh) and in the full adjustment model if they were selected for the 3-point MACE endpoint using Cox models with backward elimination, with alpha $=0.05$ required for retention. Significant variables were age, sex, race, ethnicity, $\mathrm{HbA} 1 \mathrm{c}$ New York Heart Associalion heart falure class, smoking, MI, Chronic obstruclive pulmonary disease, coronary artery disease, stroke, $\geq 50 \%$ stenosis of carolid antery, atrial ibrillation or finter, insulin trealment, ampulation, diabetic neuropathy, foot ulcer, blood pressure, heart rate, heigh body mass index, and eGFR.

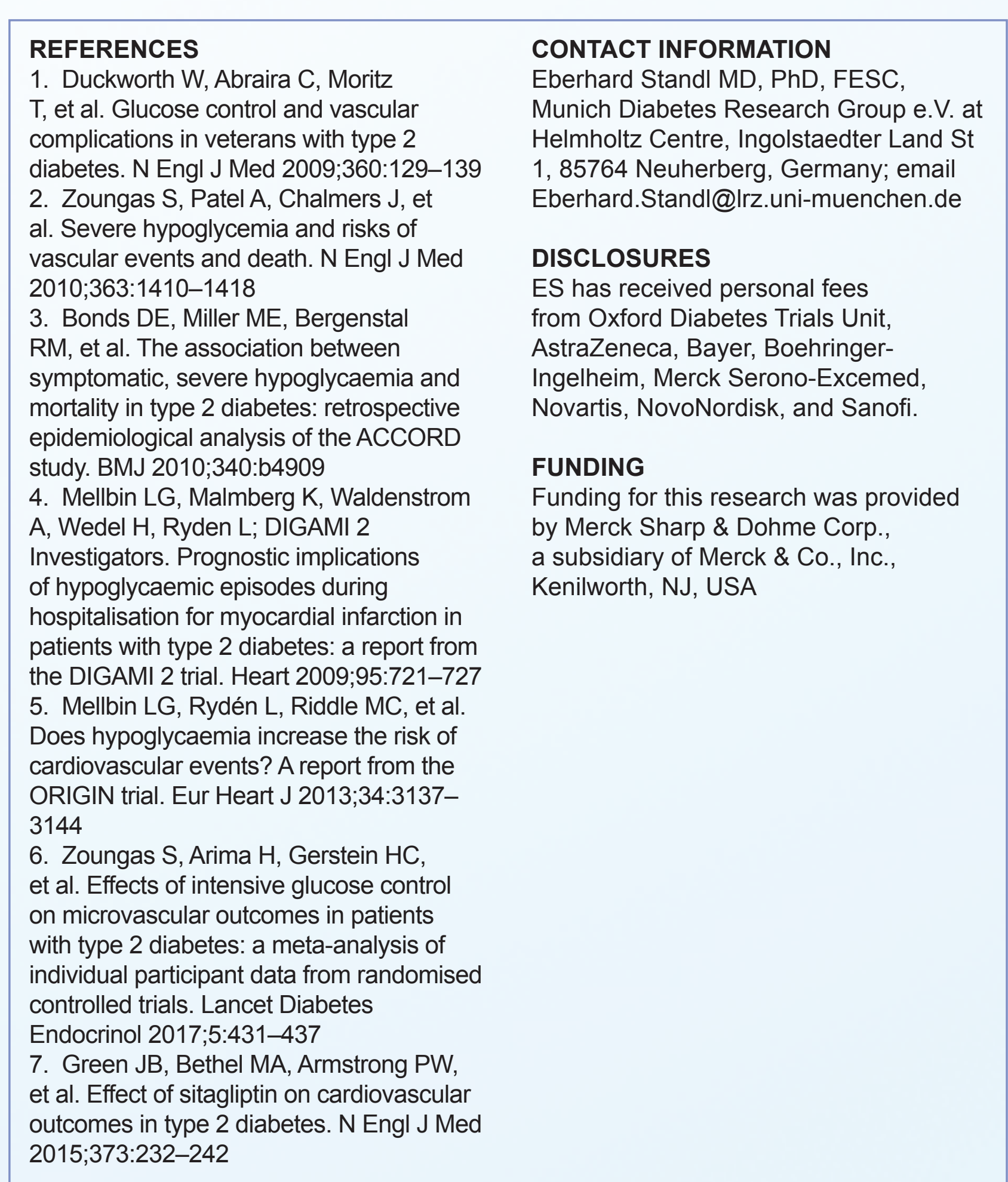
Figure 1. Time to first SHE for sitagliptin- and placebo-assigned participants (ITT
population).

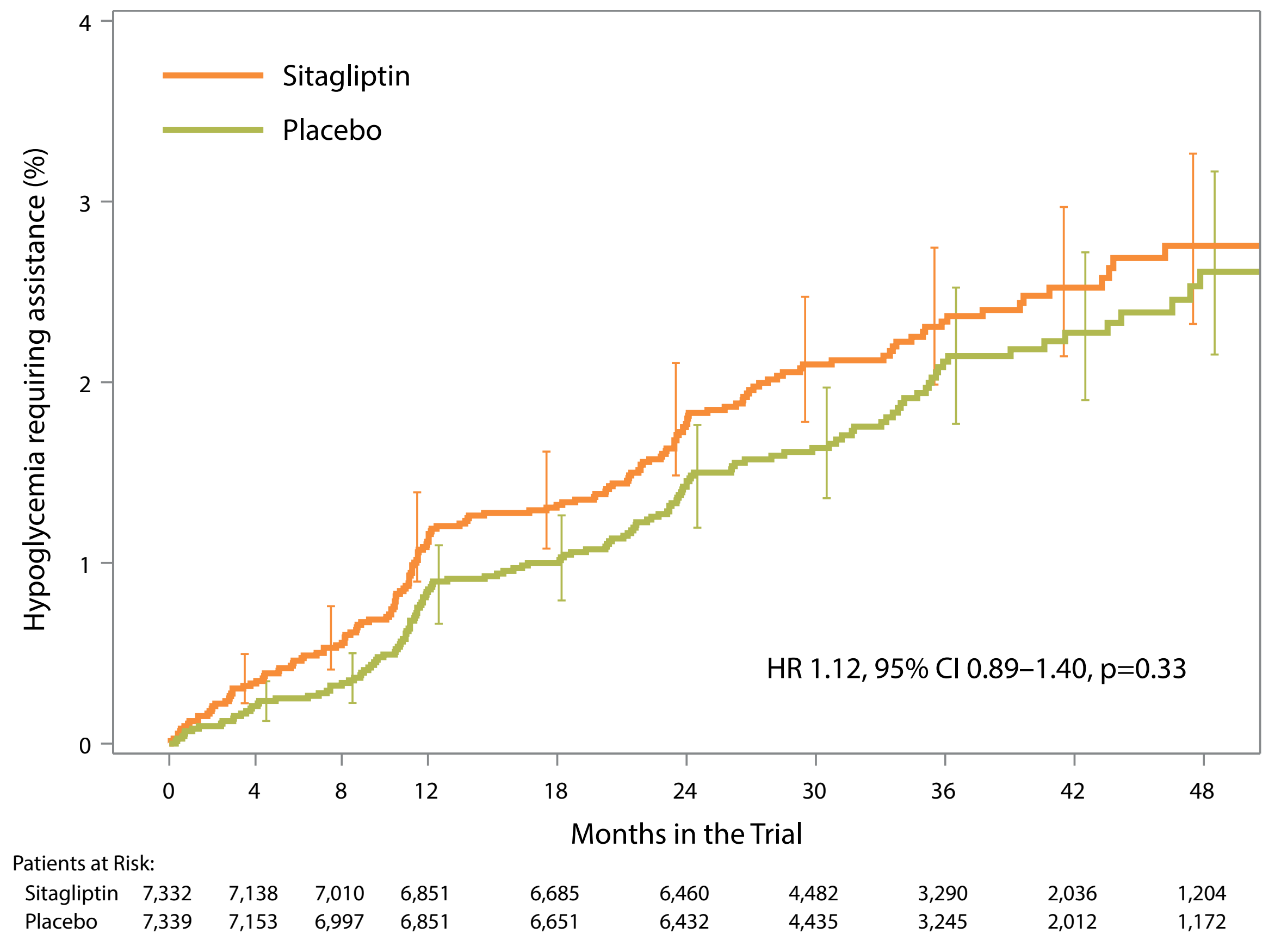

Figure 2. SHEs and subsequent risk of CV events or death. (A) Unadjusted; (B) adjusted

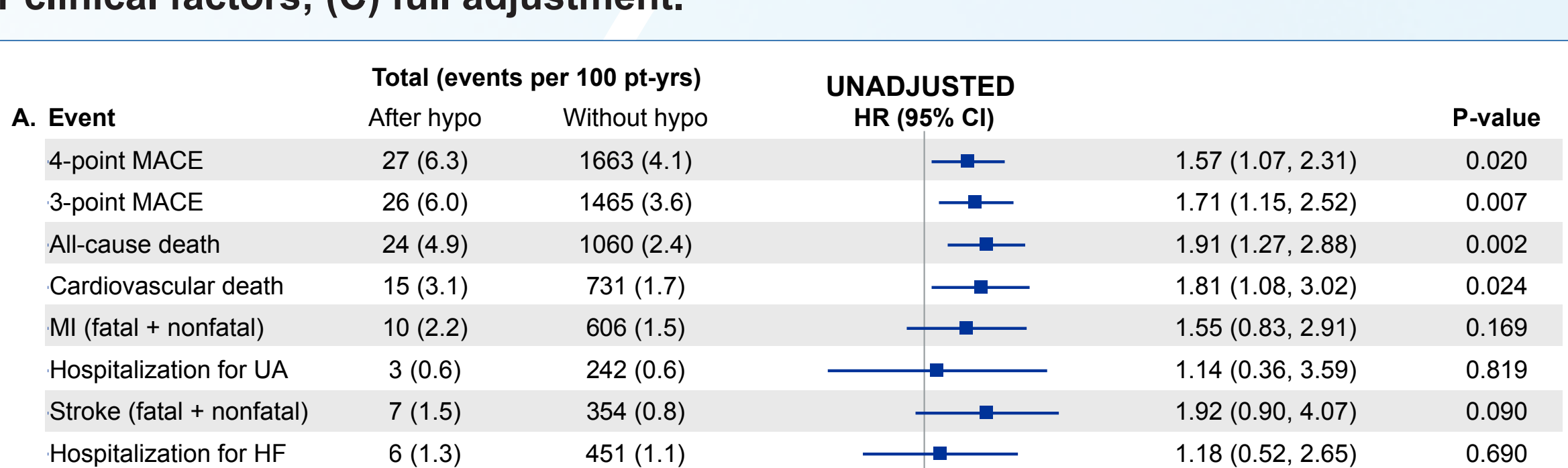
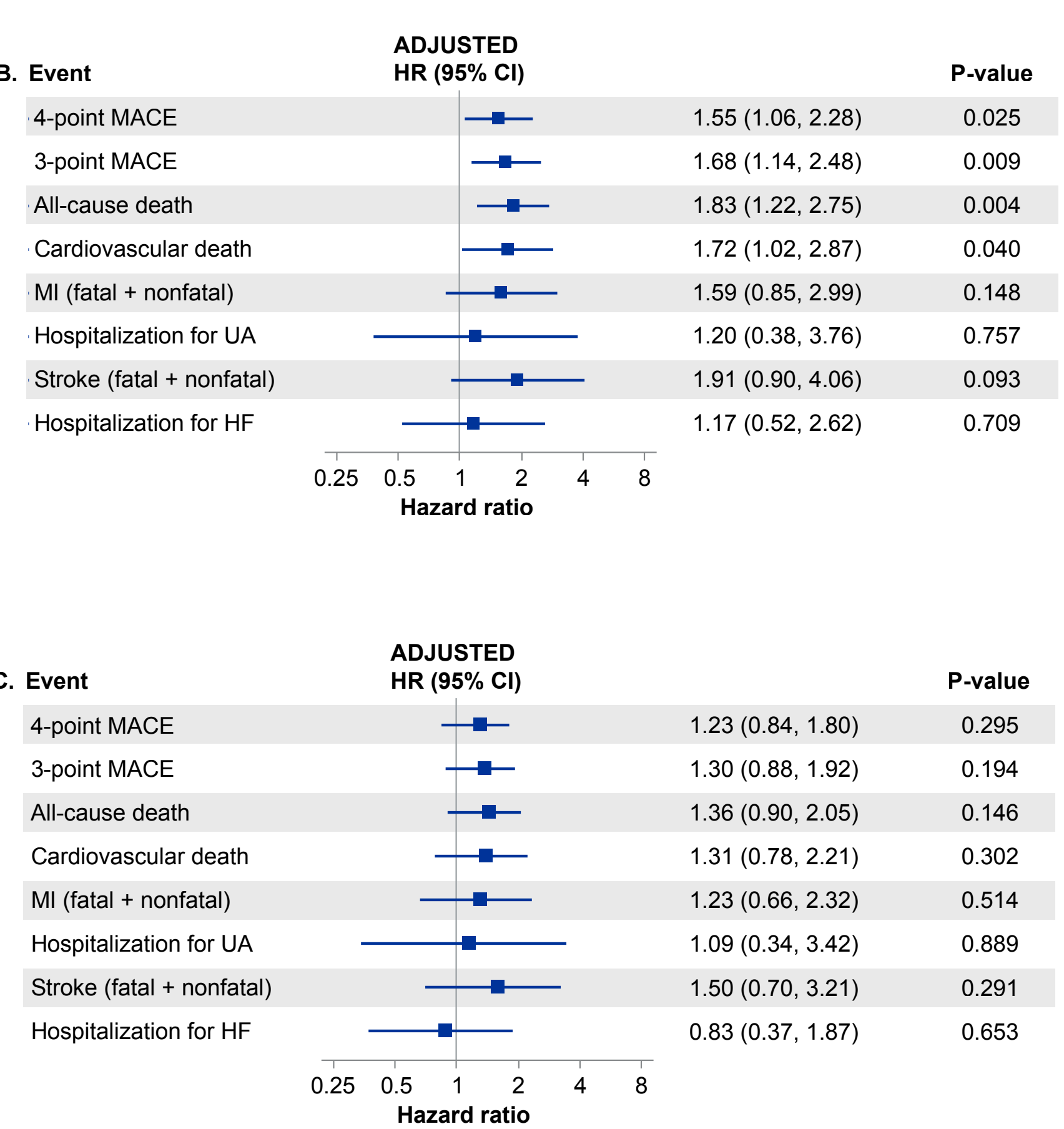

Figure 3. Nonfatal CV events or hospitalization for unstable angina (UA) or hHF and subsequent SHEs. (A) Unadjusted; (B) adjusted for clinical factors; (C) full adjustment.
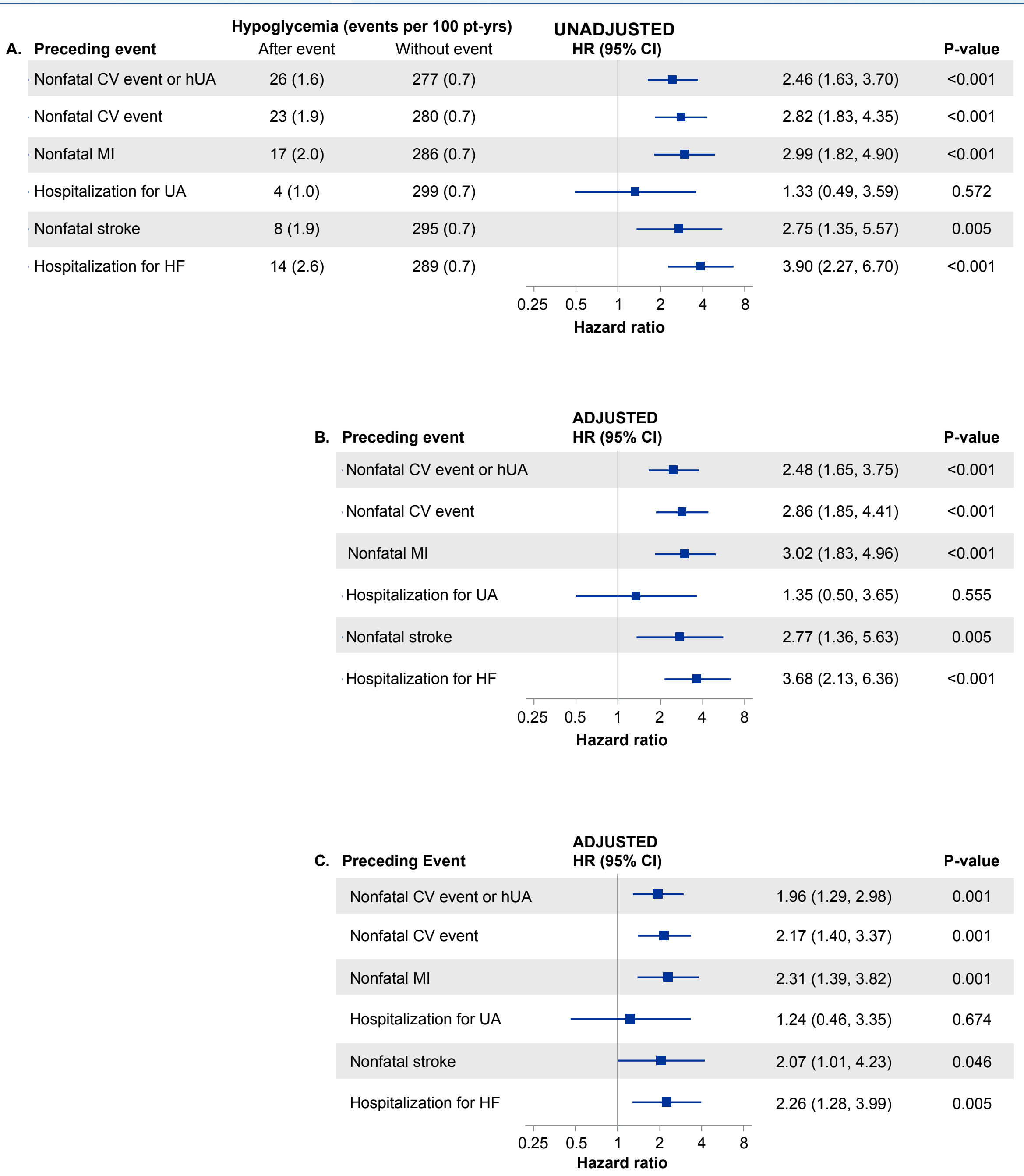

Results

SHEs were uncommon, with $303(2.1 \%)$ TECOS participants reporting one or more (7) Table 1 lists the baseline characteristics of those with and without SHEs during the trial. Participants with SHEs, compared with those without, tended to be older with longer mean duration of diabetes, lower mean body weight, and lower mean eGFR. They were also more frequently women, non-White, on insulin treatment, and more often had microalbuminuria or macroalbuminuria. Rates of reported previous $\mathrm{Ml}$ and heart failure were similar between the two groups, but participants with SHEs were more often being treated with diuretics, statins, and antithrombotic agents.

The incidence of SHEs did not differ between those assigned to the sitagliptin $(\mathrm{N}=160$ [2.2\%], 0.78 per 100 patient-years) or placebo ( $N=143$ [1.9\%], 0.70 per 100 patientyears) groups (HR 1.12, $95 \% \mathrm{Cl} 0.89-1.40, p=0.33$ ), as shown in Figure 1 . Of the 303 participants with SHEs, 68 (22.4\%) also had a 4-point MACE or hHF event. They tended to be older with higher mean body weight and lower mean eGFR, and were more often on insulin therapy or to have had an MI or heart failure with more prevalent use of betablockers, diuretics, and statins at baseline.

Of the 68 participants with SHEs and a 4-point MACE or hHF event, 34 had an SHE with a subsequent MACE or hHF event, 38 had a 4-point MACE or hHF event with a subsequent SHE, and seven had an SHE both before and after a 4-point MACE or hHF event. In three cases, participants had a single SHE and a 4-point MACE or hHF event on the same day, so which event developed first could not be determined.

Association Between SHEs and Subsequent CV or hHF Events or Death In unadjusted analyses (Figure 2A), SHEs were associated with a subsequent 4-point MACE. There was also a significant association with a subsequent 3-point MACE. After adjustment for selected clinical (demographic) factors (Figure 2B), all of these associations remained statistically significant. However, these associations all became nonsignificant after further adjustment for baseline variables tha were significantly associated with $\mathrm{CV}$ events (Figure 2C).

Association Between Nonfatal CV or hHF Events and Subsequent SHES In unadjusted analyses (Figure 3A), SHEs were associated with a previous nonfatal , honfal stroke After adjustment for clinical associations remained statistically significant after further adjustment for the wider range of baseline variables (Figure 3C)

Table 1. Baseline characteristics for participants with and without SHES (intention-to-treat population)

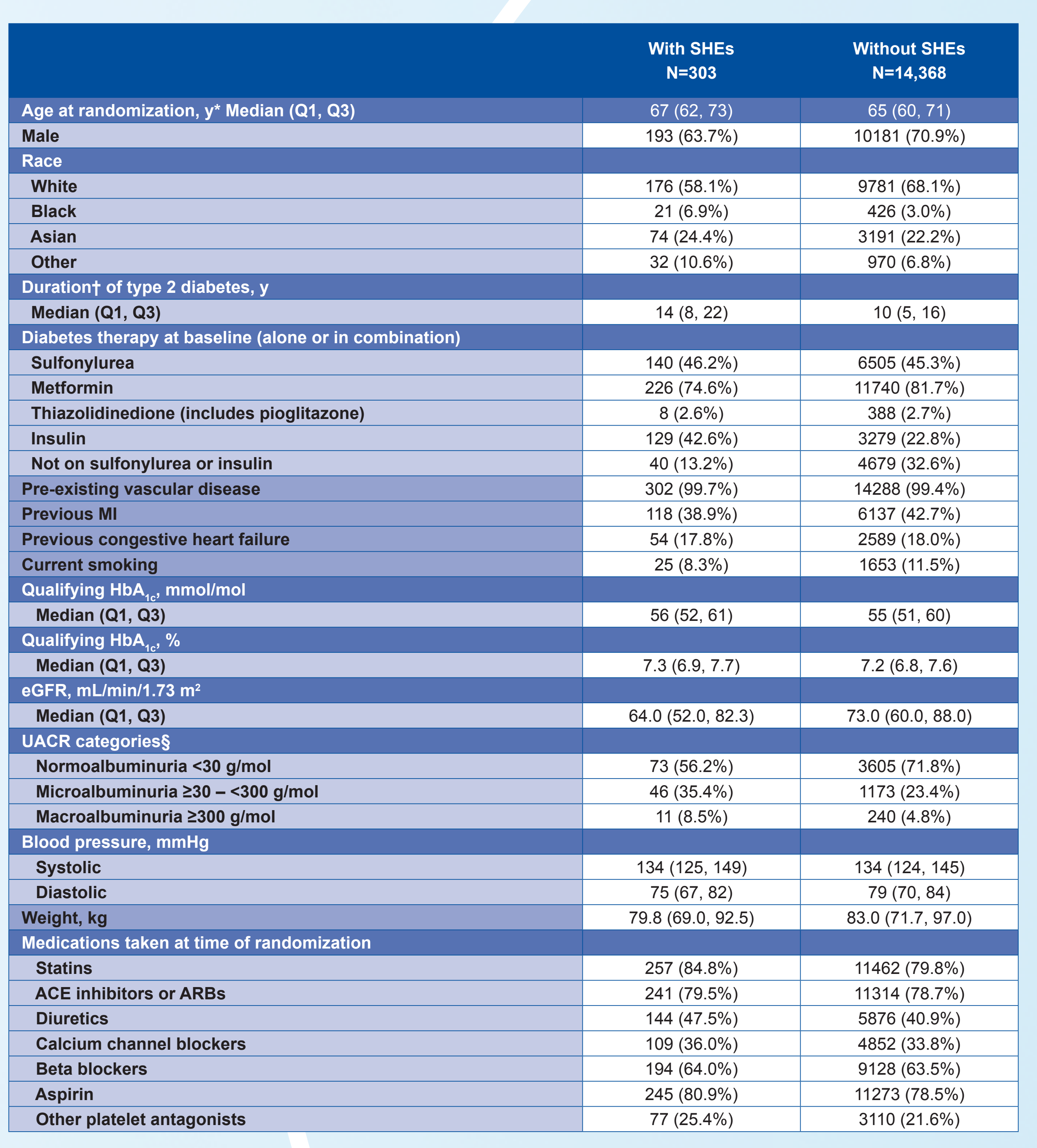

HAA $10=$ glycated hemoglob

Modification of Diet in Renal Disease (MDRD) formula was

\section{Conclusions}

Sitagliptin treatment did not increase SHE risk.

CV events were more likely following an $S H E$ and SHEs were more likely following a $\mathrm{CV}$ event, suggesting a possible common T2D 'frail' patient phenotype.

Whilst it remains critically important to seek to minimize the risk of SHEs in people with T2D, our results suggest the focus on attaining good glycaemic control to minimize the risk of diabetic complications remains a legitimate treatment goal.

A precision medicine approach may help delineate those with a frail phenotype who need special consideration from those who could benefit from lower glycaemic targets. 